\title{
Energy Efficiency in Ethernet Passive Optical Networks (EPONs): Protocol Design and Performance Evaluation
}

\author{
Yan, Ying; Dittmann, Lars
}

Published in:

Journal of Communications

Link to article, DOI:

10.4304/jcm.6.3.249-261

Publication date:

2011

Document Version

Publisher's PDF, also known as Version of record

Link back to DTU Orbit

Citation (APA):

Yan, Y., \& Dittmann, L. (2011). Energy Efficiency in Ethernet Passive Optical Networks (EPONs): Protocol Design and Performance Evaluation. Journal of Communications, 6(3), 249-261.

https://doi.org/10.4304/jcm.6.3.249-261

\section{General rights}

Copyright and moral rights for the publications made accessible in the public portal are retained by the authors and/or other copyright owners and it is a condition of accessing publications that users recognise and abide by the legal requirements associated with these rights.

- Users may download and print one copy of any publication from the public portal for the purpose of private study or research.

- You may not further distribute the material or use it for any profit-making activity or commercial gain

- You may freely distribute the URL identifying the publication in the public portal

If you believe that this document breaches copyright please contact us providing details, and we will remove access to the work immediately and investigate your claim. 


\title{
Energy Efficiency in Ethernet Passive Optical Networks (EPONs): Protocol Design and Performance Evaluation
}

\author{
Ying Yan and Lars Dittmann \\ Department of Photonics Engineering, Technical University of Denmark, Kgs. Lyngby, 2800 Denmark \\ Email: \{yiya, ladit\}@ fotonik.dtu.dk
}

\begin{abstract}
As concerns about energy consumption grow, the power consumption of the EPON becomes a matter of increasing importance. In respect of energy efficiency, the current standard has no management protocols aiming to reduce power consumption in EPONs. In this paper, we propose an Energy Management Mechanism (EMM) for downlink EPON systems. The proposed mechanism is designed to enhance the standardized control scheme in EPON with the objective to increase energy efficiency while satisfying diverse QoS requirements. The main idea is to put an Optical Network Unit (ONU) into the sleep mode and determine a suitable wakeup time scheduler at the Optical Line Terminal (OLT). A generic EPON system is considered, which is composed of an OLT and several ONUs that are EMM enabled. An energy consumption optimization problem aimed at saving energy is proposed and two heuristic sleep mode scheduling policies are addressed to solve it. The scheduling algorithms are tightly coupled with the upstream bandwidth allocation and downstream transmission scheduling together through an integrated approach in which awake time in ONUs is minimized. There is a trade-off decision between maximizing the power saving and guaranteeing the network performance at the same time. Simulation results show that an EMM-based EPON with well designed scheduling disciplines is essential to achieving significant energy saving while meeting the delay constraint.
\end{abstract}

Index Terms-EPON, Energy efficiency, MPCP, scheduling algorithms.

\section{INTRODUCTION}

It has been widely recognized that reducing power consumption in data communication network becomes an important issue to global environment and future human life [1]-[5]. Recent research has shown that, among all network segments, broadband access networks are the highest share of the network energy consumption, which consumes more than 75 percent of energy consumption by all telecommunication equipments today. Due to the rapid expansion of access network connectivity, increasing number of users and data rate are foreseeable in the future broadband access networks. As a result, energy consumption of future broadband access networks would

Manuscript received Feb 22, 2011; accepted Mar 11, 2011.

This paper is based on "Energy Efficient Ethernet Passive Optical Network (EPONs) in Access Networks," by Ying Yan and Lars Dittmann, which appeared in the conference proceedings for the 10th WSEAS International Conference on Applied Informatics And Communications (AIC) in Taipei, Taiwan August, 2010 continue to rise, with a steady annual growth rate in the near future. Therefore, it has raised attention in both academia and industry to design energy efficient network systems. Commonly, sleep mode operation is introduced to allow that nodes (or stations) can switch to sleep when they are idle and wake up when they receive or transmit packets. Such approach has been widely exploited in wireless networks, where saving battery power in mobile stations is of paramount importance due to finite power supplies in wireless devices [6]-[11].

The EPON, a prevailing deployed broadband access technology, provides cost efficiency and high data rate for the last mile access. A typical EPON is a Point-toMultipoint (PMP) network with a tree based topology, where an OLT connects multiple ONUs via optical links. The OLT plays a role of distributor, arbitrator and aggregator of traffic. In the upstream direction (from ONUs to the OLT), multiple ONUs share a single link and traffic may collide. The OLT distributes the fiber capacity using an upstream bandwidth arbitration mechanism to avoid collisions. In the downstream direction (from the OLT to ONUs), data frames are broadcasted to all ONUs. ONUs filter and accept data that are addressed to them. However, ONUs have to constantly listen and examine downstream traffic, which results in wasting significant energy in the ONU. Shown in Fig. 1, in the traditional EPON, ONUs consume energy to keep active when there is neither upstream nor downstream traffic. As a result, minimizing power consumption is a major factor driving the design of EPON devices and of the protocols therein. An effective Energy Management Mechanism (EMM) that schedules the sleep mode period to ONUs is a key to conserve power.

The major sources of energy waste of shared resource EPON system include: overhearing, control packet overhead, and idle listening. Overhearing represents that an ONU receives and decodes packets that are not destined to it. Control packet overhead means the transmission of control messages, which are necessary to coordinate transmission between the OLT and ONUs, and unfortunately increases time and energy consumption. At last, idle listening means active time and energy ONUs spent on waiting for the next upstream and downstream transmission. It is clear that an energy efficient scheduler is designed aiming to eliminate these sources of useless 


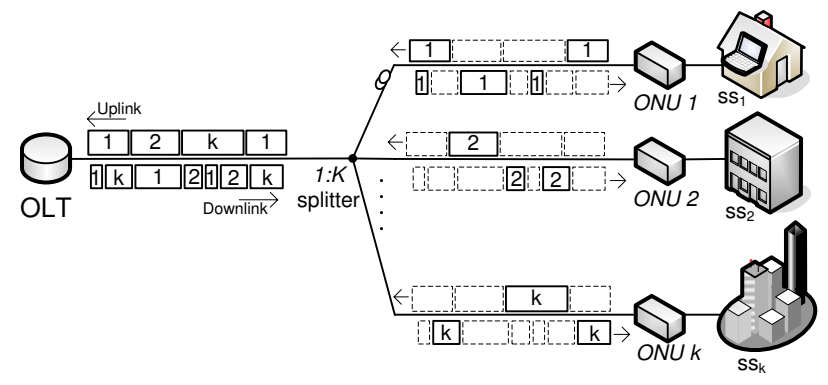

Figure 1. EPON upstream (multi-point to point) and downstream (point to multi-point) transmission.

energy consumption.

To reduce power consumption, ONUs are designed to enter sleep mode when they do not need to either receive or send traffic. During a sleep period, ONUs turn off the transceiver in order to save energy. In case there is incoming data for a sleeping ONU, data is queued in buffers at the OLT. Obviously, it is better to keep an ONU to stay in sleep mode as much as possible to conserve its energy. However, a power management mechanism with efficient scheduling for sleep and wake-up periods among multiple ONUs is a challenging task due to:

- ONUs should wake up and exchange traffic with the OLT. However, arrival traffic profile is not always known by the device, because the downstream and upstream traffic can be either periodically or aperiodically arrived. Therefore, the sleep period should be carefully assigned in order to avoid missing any incoming packets.

- During sleep period, data is buffered and prescheduled for upstream and downstream transmission in both the OLT and ONUs. Due to QoS constraints in terms of delay and latency, the sleep period should be carefully scheduled, so target ONUs can wake up and complete transmission without violating QoS requirements.

As discussed in next Section II, several studies have been proposed to analyze the power consumption for EPON, while applying the approach of allowing ONUs to sleep mode. Although Different aspects have been investigated on how to minimize energy consumption in PON systems, there have been few studies focusing on the protocol design for supporting sleep mode ONUs in EPON. To implement EMM in EPON, the legacy control scheme requires modifications and extensions. Moreover, very few have explicitly addressed the trade-off between energy consumption and network performances. To the best of our knowledge, our work is the first to propose the assignment of sleep period to ONUs taking into account of the traffic transmission in both upstream and downstream directions.

The remainder of this paper is organized as follows. We first give a brief description of related works in Section II. The introduction of the system model is addressed in Section III. The design of EMM based EPON with two different downstream schedulers is introduced in Section IV. Simulation environments and results are outlined and discussed in Section V. Finally, conclusions and future work are drawn in Section VI.

\section{RELATED WORKS}

Issues of energy efficiency have been studied in EPONs as well as in other optical access networks. In this section, we present related works and compare their models with ours.

Authors in [12] propose a management protocol for ONUs to request entering in sleep mode. The OLT reserve a minimal bandwidth for ONUs in sleep mode. During a sleep period, no connection is established and no traffic is delivered. The sleep mode can be terminated by either the OLT or the ONU by using an administration message. This protocol eliminates conventional requirements for a complete activation procedure in ONUs. The authors address the impact of sleep mode protocol for re-activation process in GPON.

In [13], the authors present implementation challenges of sleep mode operation in PONs, such as achieving clock synchronization and reducing the clock recovery overhead. The process of regaining network synchronization after waking up from sleep mode is studied in both GPON and EPON system. Two novel ONU architectures are proposed and compared with current ONU architectures on power consumption and wakeup overhead. As already indicated, we focus on protocol design and do not address the physical implementation issues in our work. The architectures as well as the overhead values proposed in [13] can be used for this purpose.

Another paper [14] proposes a power saving mechanism for 10 Gigabit class PON, where ONUs are implemented with a sleep and periodic wakeup regime. The sleep mode is triggered based on negotiations between the OLT and the ONU, and a variable sleep time is allowed. The authors use a prediction model of traffic profiles to determine the initiation and the duration of sleep mode. A probabilistic analysis of the presence or absence of downstream traffic is required in order to ensure the ONU can wake up and the packet is delivered on time. This model differs from ours in that our model does not use estimation and the energy management mechanism is mapped in EPON MAC layer.

Author in [15] presents the EPON power saving issue on the IEEE 802.3az meeting in September 2008. The functions for power saving are proposed to be implemented in both the OLT and ONUs. The initiation and termination of sleep mode by using handshake messages are drafted. The attention of energy efficiency is raised in the IEEE standardization, however, there are considerably few research works in saving energy consumption of optical network management systems. Our work investigates the current control mechanism for EPON and proposes control protocol for sleep mode operation.

The relationship between energy and data rate in general optical IP networks was also studied in a different context. For example, [16] presents power saving of 
different network architectures, including all-optical networks, first-generation networks and multi-hop networks. Power consumption of electronic versus optical components in optical WDM networks is investigated under different traffic loads. In [4] this problem is generalized by considering the power consumption in an optical IP network. A new network-based model is established, which includes core, metro and access networks.

\section{SySTEM MODEL}

In this paper, we consider an EPON system consisting of an OLT, $1: K$ splitter and multiple ONUs. The upstream and downstream traffic are separated in different wavelength, typically $1310 \mathrm{~nm}$ for the upstream transmission and $1550 \mathrm{~nm}$ for the downstream transmission. TDMA is used in the physical layer where bandwidth is divided in time slots. Each ONU maintains an upstream buffer and sends upstream data to the OLT during assigned time slots. For simplicity, we assume in this paper that the upstream data rate is fixed for all ONUs. In the downstream direction, when the arrival rate at the OLT exceeds the output data rate, data are queued in the OLT and transmitted when downstream bandwidth is available.

We also assume that the OLT and ONUs can regain synchronization successfully so that ONUs can enter sleep mode and return back to awake mode. The power consumption value in the awake state includes energy consumed in listening to the OLT and in receiving or transmitting data. We neglect the transition delays and the energy consumption when an ONU changes states. We assume that the packets are able to be served within an integer number of time slots. During the observation period, the number of ONUs is also fixed. Next we list some notations (Table I) used in the rest of this work and then formulate the energy saving scheduling problem.

Assuming that the overall transmission period is divided into $N$ time slots, ONUs are either in awake mode

\begin{tabular}{|c|c|}
\hline Notations & Value \\
\hline$K$ & The number of ONUs registered in the OLT. \\
\hline$N$ & $\begin{array}{c}\text { The total time slots within the overall observed } \\
\text { transmission period. }\end{array}$ \\
\hline$n$ & $\begin{array}{c}\text { The index of time slot. } n \in N \\
s_{i}^{n}\end{array}$ \\
\hline$T_{i}^{n}$ & $\begin{array}{c}\text { ONU } i \text { state indication (awake mode } \\
\text { or sleep mode) in time slot } n .\end{array}$ \\
\hline$P_{\text {awake }}$ & $\begin{array}{c}\text { Period of time slot } n \text { in ONU } i . \\
\text { The average amount of energy (Watt) } \\
\text { consumed during the awake period. }\end{array}$ \\
\hline$P_{\text {sleep }}$ & $\begin{array}{c}\text { The average amount of energy (Watt) } \\
\text { consumed during the sleep period. }\end{array}$ \\
\hline
\end{tabular}

TABLE I.

NOTATIONS IN ENERGY EFFICIENCY MECHANISM or in sleep mode during the time slot $n$. The total energy expenditure model is formalized as follows:

$$
\mathbf{E}=\sum_{i=1}^{K} \sum_{n=1}^{N} T_{i}^{n} \cdot\left[s_{i}^{n} \cdot P_{\text {awake }}+\left(1-s_{i}^{n}\right) \cdot P_{\text {sleep }}\right]
$$

\section{A. Analysis of Sleep Mode Operations}

Within a period, the total energy consumption includes energy consumed by $M$ ONUs, which are in active state. As expressed in our energy expenditure model (Equation 1), the total energy consumed by each ONU consists of two parts: in either the awake or sleep mode. In the awake mode, there are three different states: receiving packets, sending packets and idle listening. The idle state is defined as the time when an ONU is active without any receiving or transmitting action. In the sleep state, ONUs switch off transceiver functions and keep a timer on to count down the wakeup time. Nodes consume much less power in the sleep mode than in the awake mode. For instance, regarding to different architectures for a sleep mode enabled ONU [4], the expected power consumptions require $2.85 \mathrm{~W}$ for active and $750 \mathrm{~mW}-1.28 \mathrm{~W}$ for sleeping. When ONUs enter sleep state, ONUs are in the absence of traffic at the optical transmission interface. However, some functions, such as clock and data recovery and back-end digital circuit, are still powered on. In this sense, the sleep mode differs from power down, which is usually an indication of any equipment fault or disconnection in EPON. If an ONU is reported as deactivation after a power down, the ONU has to register itself to the OLT again via the periodic discovery processing. On the other hand, using the sleeping mode, ONUs can wake up, listen to, and communicate with the OLT without the registration process, when the sleep period is finished.

As shown in Fig. 2, a timer is utilized in the sleep mode control. Upon receiving notification of entering the sleep mode, the timer counts down and the ONU wakes up when the timer is expired. When an ONU wakes up from the sleep mode, there is an overhead time window $\left(T_{o v}\right)$ for recovering the OLT clock $\left(T_{\text {recv }}\right)$ and retrieving network synchronization $\left(T_{\text {sync }}\right)$. The clock recovery time is various in different implementations of ONU receiver architectures. For synchronization process, a fixed Start Position Delimiter (SPD) is embedded in the EPON control message header, which allows ONU to gain synchronization with the start of the EPON frame. The synchronization time in EPON is up to $125 \mu s$ [13].

Following our model, it is obvious that energy depletion is determined by the time the ONU spend in awake mode. An optimal EMM solution is to schedule ONUs into sleep mode whenever there is no transmission in order to minimize the overall power consumption. However, since there are QoS requirements in terms of delay and throughput, it requires a scheduling policy to improve energy efficiency without violating the QoS requirements. Therefore, this work deals with the trade-off between the allocation of wakeup frequency and the queuing delay in the EPON. 


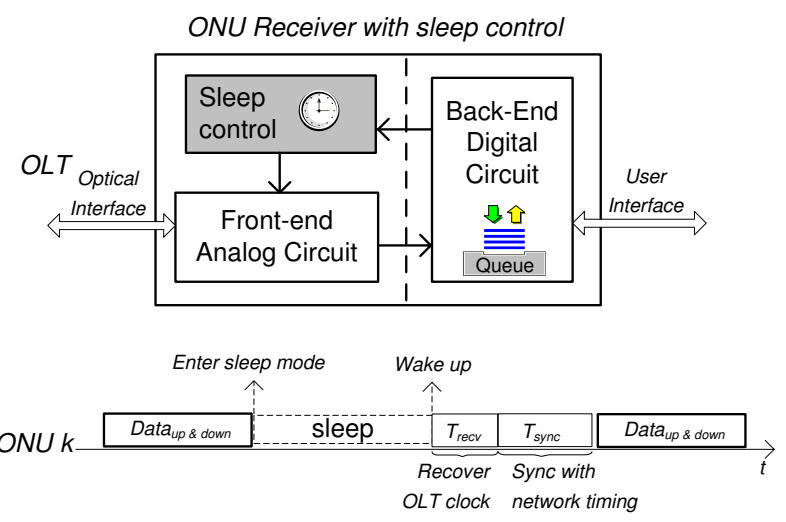

Figure 2. ONU receiver with sleep control function.

\section{B. Analysis of Energy Consumption and Delay Perfor- mance}

When energy management mechanism is implemented in EPON, ONUs turn their optical communication interface on and off to minimize energy consumption. Therefore, in order for an ONU to communicate with the OLT, it must be in active mode. As a centralized control station, the OLT schedules active duty cycles and informs connected ONUs about its assignments. An ONU must comply with the noticed sleep period and wakeup time. When an ONU is in sleep mode, the downstream traffic destined to the ONU is queued in the OLT. This communication model imposes a clear trade-off between the delay encountered by a packet and the time during which ONUs are in active mode. Solutions for addressing this trade-off depend, to a large extent, on the following aspects:

1) The expected amount of data to be received and transmitted.

2) The polling scheme and upstream bandwidth allocation scheme: for example, the sequence of polled ONU and the size of bandwidth granted to the ONU.

3) Whether the OLT process the packets and send to ONUs with priority discrimination.

With respect to 1 , if the data arrival rate is high and continuously delivered, data are queued and the OLT can schedule transmission. With respect to 2 , if the polling scheme and upstream bandwidth allocation are flexible enough, it can determine the order of ONUs to communication according to the expected delay constraint. With respect to 3 , if the OLT can process packets, it may buffer and merge several packets for aggregation.

We first formulate our scheduling problem: find an optimal scheduling discipline that minimizing the average energy consumption of EPONs while guaranteeing an upper bound on the queuing delay. This problem is formalized as an optimization problem that an optimal assignment of wakeup time to ONUs is an assignment that guarantees an upper bound $D_{\max }$ on the maximum delay while minimizing the total energy spent by the ONUs in awake mode. Let $K$ be a set of ONUs that are awake and communicate with the OLT during the whole $N$ observation periods. Let $T_{i}^{n}$ and $P_{\text {awake }}$ be the awake period and the average energy consumption for each ONU $i$, respectively. Then, the goal is to:

$$
\text { minimize : } \quad E=\frac{1}{K} \sum_{i=1}^{K} \sum_{j=1}^{N} T_{i}^{j} \cdot P_{\text {awake }}
$$

subject to:

$$
d_{i}^{m}<D_{\max }
$$

where $d_{i}^{j}$ is the delay experienced by every connections in AG $i$, which should deliver qualified services.

\section{Energy Management Mechanism (EMM)}

In this section, we introduce the implementation of EMM together with EPON MAC protocol, Multipoint Control Protocol (MPCP) [17]. The OLT is in full control of the bandwidth allocation in both downstream and upstream. An ONU can associate with the OLT either in normal mode, referred to as Power Ignoring Scheme (PIS), or in EMM mode. When an ONU is in normal mode, it constantly stays awake and consumes power, i.e., never goes into sleep mode. In the following, we assume that all ONUs are EMM-enabled.

In order to minimize power consumption, ONUs remain in a sleep mode most of the time while adhering to the assignment from the OLT. We show how being in EMM yields considerable power saving for an ONU as compared to PIS case. In addition, we introduce how the sleep mode scheduling scheme has an impact on the energy and delay performance of the EMM ONUs. The sleep period and wakeup time can be calculated and assigned using either an Upstream Centric Scheduling (UCS) algorithm or a Downstream Centric Scheduling (DCS) algorithm.

\section{A. Energy Efficient Scheduler Design}

In EPONs, the downstream and the upstream transmission are separated, and the OLT plays a central role to reserve bandwidth and to serve traffic transmission. In this paper, a subframe period (SP), $T_{s p}$, refers to the time assigned to an ONU to send upstream data to the OLT (SP-UL) or to receive downstream data from the OLT (SP-DL). In this work, we will analyze the energy consumption and determine the wakeup time by considering both the upstream and downstream data since the instants of terminating sleep mode by upstream and downstream transmission are different.

- Downstream bandwidth allocation: In the MPCP specified in IEEE 802.3ah, the OLT broadcasts a downstream packet with destination MAC address to all ONUs. ONUs that are awake to listen if the OLT has packets to deliver to them check the header for destination identification. Since there is no retransmission mechanism in the EPON downstream, it takes a long latency if the ONU misses 
packets. Therefore, ONUs keep active all the time in order to listen to the OLT and receive packets if the destination identification is matched. Since the power saving mechanism is ignored traditionally, time and energy are wasted when an ONU is active to listening to the OLT and decoding packets destined to other ONUs.

- Upstream bandwidth allocation In the MPCP, the OLT maintains a polling list and a polled ONU has the right to transmit data within its assigned upstream time slots. The OLT allocates upstream time slots to each ONU in order to avoid that multiple ONUs transmit to the uplink simultaneously. The upstream bandwidth information such as the start time and the length of granted transmission window is carried in the GATE message. The OLT polls each ONU by sending them GATE message and ONUs transmits traffic within the assigned upstream time slots after receiving the GATE message. The upstream bandwidth is distributed among all ONUs by using either a fixed allocation scheme or a dynamic bandwidth allocation (DBA) scheme [18]. In the former case, each ONU is scheduled with a fixed amount of upstream transmission period. In the latter case, the bandwidth is allocated dynamically with a bounded size according to ONUs' request. A cycle $\left(T_{\text {cycle }}\right)$ refers to an interval between two successive polling messages to an ONU. Since the power saving mechanism is ignored traditionally, the time is wasted when an ONU is active to waiting for its next upstream transmission period.

The EPON supports energy management at the MAC layer, and ONUs need to negotiate with the OLT in order to decide the power saving parameters. The power saving parameters include the time to sleep and wake up. Theoretically, the power saving control can be initiated either at the OLT or at the ONUs, if they are in an idle state, where no packet to be received or sent. However, as the ONU is an aggregation node that collects traffic from low level networks and relays to the OLT, it is commonly assumed that ONUs always have queued data for upstream transmission. In this work, we consider only the OLT triggered power saving mechanism. We focus on protocol design of EMM and the scheduling schemes to maximize power saving subject to the constraint of packet delay.

With respect to bandwidth allocating, since the MPCP is central control protocol, the OLT has full knowledge to assign transmission bandwidth in both upstream and downstream directions. This implies that the order of granted ONUs and the amount of granted bandwidth are determined by the OLT. An intuitive solution for EMM scheduler is to aggregate the downstream traffic in order to minimize the time allocated to ONUs for idle states. The general design of EMM based EPON proposes following functions in the OLT and ONUs:

\section{1) OLT operation:}

The OLT can buffer downstream traffic and sched- ule the appropriate transmission period to each ONU. To meet the QoS requirement of delay sensitive traffic, the downstream bandwidth is allocated such that every packet can be served before its deadline. The original control message GATE is modified with additional fields indicating the assigned sleep time and wakeup time. To assign the wakeup time for the next upstream transmission, the OLT needs to calculate the time for an ONU to upload and download data packets. Considering the wakeup time to transmit the upstream packet, the OLT determines and allocates the upstream transmission windows for all ONUs. As for the wakeup time to receive the next downstream packet, since the number of buffered downstream data is completely know by the OLT, it can calculate and schedule downstream transmission.

\section{2) ONU operation:}

After scheduling the sleep period, the OLT sends a control message to the ONU for the permission to transit into sleep mode. Upon receiving this message with parameters start time for sleep and wakeup time from sleep, the ONU enters into sleep mode. After a sleep mode, the ONU transits back to the awake mode again. Scheduled ONUs should wake up according to the assigned wakeup time and check the sleep-mode GATE message $\left(G_{s}\right)$. Upon receiving the $G_{s}$ message, the ONU derives the sleep period and obeys the assigned upstream and downstream bandwidth allocation. As for the upstream subframe period announcement, the assigned wakeup time implies its next access time to the upstream transmission. For a $G_{s}$ message announcing the downstream subframe period, the ONU should awake at the notified wakeup time to receive the buffered data and then back to sleep afterwards.

\section{B. Upstream Centric Scheduling (UCS) Algorithm}

The main idea of UCS scheme is that the OLT assigns the awake period to ONUs according to their corresponding upstream allocation. The OLT grants the upstream bandwidth by polling each ONU. During the granted upstream subframe period, the ONU is awake. Once an ONU transits into the awake state, the OLT with UCS algorithm only transmits downstream packets to the awake ONU, and queues those packets destined for 'sleep' ONUs into a buffer.

In this subsection, we give a description of the UCS algorithm. For simplicity of illustration, we consider a system of an OLT and three ONUs for two upstream transmission periods. For more ONUs and transmission periods, the same logic can be applied. Fig. 3 shows the process of UCS protocol. In this work, the $t$ denotes for the instant time and $T$ represents for a period. The OLT maintains an entry table, which contains 1) the start transmission time $\left(t_{\text {start }}^{n}\right)$ and granted bandwidth $\left(B W_{i}^{n}\right)$ for allocating upstream bandwidth; 2) the sleep 
time $\left(t_{\text {sleep }_{i}}^{n}\right)$ and the wakeup time $\left(t_{\text {wake }}^{n}\right)$ for assigning sleep period. The table is updated every cycle $n$ for $O N U_{i}$. Downlink data are stored in subqueues for each ONU.

As shown in Fig. 3, the $O N U_{1}$ wakes up at $t_{0}$ and receives sleep mode GATE message $\left(G_{s 1}^{1}\right)$ at the time $t_{1}$. After learning the granted upstream subframe period $\left(T_{s p-u l}\right), O N U_{1}$ generates REPORT message $\left(R_{1}\right)$ and starts upstream transmission $\left(d_{u l}^{1}\right)$ complying with the assignment. During $T_{s p-u l} 1$, the OLT derives downstream data $\left(d_{d l}{ }_{1}^{1}\right)$ from the subqueue for the $O N U_{1}$ and transmits them to the $O N U_{1}$. Notice that the data $d_{d l}$ represents either one data packet or a series of integrated data packets. The start time of sleep mode $\left(t_{\text {sleep }} 1\right)$ for $O N U_{1}$ is assigned so that the $O N U_{1}$ enters into sleep mode immediately when the upstream subframe period is completed at $t_{2}$. The calculations of upstream transmission period and the time to turn into sleep mode are listed in Equation 4 and Equation 5.

$$
\begin{gathered}
T_{s p-u l, i}^{n}=\frac{B W_{i}^{n}}{R_{o}}, \quad i \in K, n \in N \\
t_{\text {sleep }, i}^{n}=t_{\text {start }, i}^{n}+T_{s p-u l, i}^{n}, \quad i \in K, n \in N
\end{gathered}
$$

where $B W_{i}^{n}$ is the allocated upstream bandwidth for the $i^{t h}$ ONU during the $n^{t h}$ cycle. $R_{o}$ is the transmission rate of the optical upstream and $T_{g}$ is the guard time between two successive upstream transmissions. The wakeup time $\left(t_{\text {wakeup }} 1\right)$, which is same as the length of sleep period, is calculated based on the period before the $O N U_{1}$ is polled again $\left(T_{\text {cycle }}^{1}\right)$. In the Fig. 3 , the next wakeup time for the $O N U_{1}$ is $t_{3}$. The value of wakeup time is computed at the OLT and assigned to each ONU. The polling cycle is computed using Equation 6 .

$$
\begin{aligned}
T_{\text {cycle }, i}^{n} & =\sum_{i=1}^{K}\left(T_{s p-u l, i}^{n}+T_{g}\right) \\
& =\sum_{i=1}^{K}\left(\frac{B W_{i}^{n}}{R_{o}}+T_{g}\right), \quad i \in K, n \in N
\end{aligned}
$$

After a period of $T_{\text {cycle }}$, the interval between two adjacent polling messages, an ONU is polled again at $t_{4}$ $\left(t_{1}+T_{\text {cycle }} 1\right)$. The calculation of ONU wakeup time needs to take the overhead period into account due to the time of recovering the OLT clock and retrieving the network synchronization. The computation of the overhead period $\left(T_{o v}\right)$ and the time to wake up are listed as Equation 7 and Equation 8, respectively.

$$
\begin{gathered}
T_{o v}=T_{\text {recv }}+T_{\text {sync }} \\
t_{\text {wakeup }, i}^{n}=t_{\text {start }, i}^{n}+T_{\text {cycle }, i}^{n}-T_{\text {ov }}, \quad i \in K, n \in N
\end{gathered}
$$

During the observation period, the total awake time for each ONU is the sum of allocated upstream time slots and an ONU enters the sleep mode otherwise. During the subframe period, the $O N U_{i}$ transmits upstream data $d_{u l_{i}}{ }^{n}$

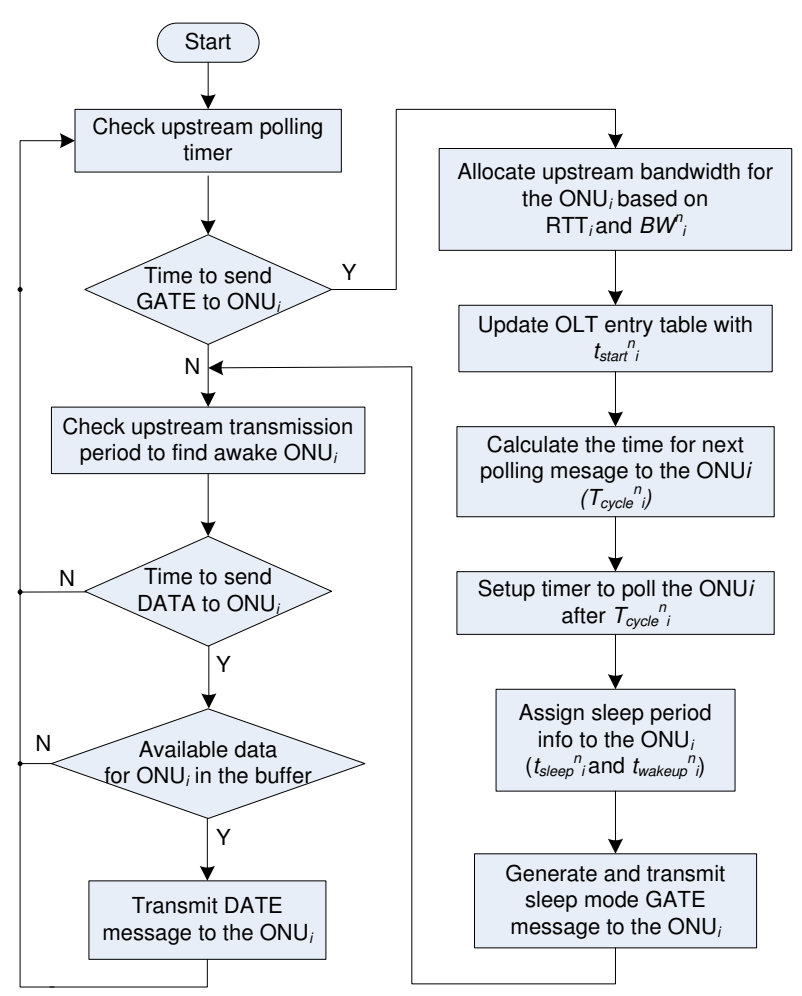

Figure 4. Flowchart of the UCS algorithm designed in the OLT.

and receives downstream data $d_{d l}{ }_{i}$, within the granted time slot $n$. Notice that the size of the uploaded and downloaded data must equal or less than the upstream bandwidth, i.e. $\operatorname{size}\left(d_{u l_{i}}^{n}\right) \leq B W_{i}^{n}$ and $\operatorname{size}\left(d_{d l_{i}}^{n}\right) \leq B W_{i}^{n}$. The total energy consumed by the ONU $i$ is illustrated in Equation 9:

$$
\begin{aligned}
\boldsymbol{E}_{\mathbf{U C S}} & =\sum_{i=1}^{K} \sum_{n=1}^{N}\left[T_{s p-u l, i}^{n}+T_{o v}\right] \cdot P_{\text {awake }} \\
& +\sum_{i=1}^{K} \sum_{n=1}^{N}\left[T_{c y c l e, i}^{n}-T_{s p-u l, i}^{n}-T_{o v}\right] \cdot P_{\text {sleep }}
\end{aligned}
$$

The UCS based scheduler is simple because the sleep period is determined based on the upstream transmission. An OLT with the UCS based scheduler uses the awake ONU, which is assigned to the upstream transmission as destination to retrieve buffered data. The algorithm is presented in the flowchart in Fig. 4. However, the performance of downstream data latency and bandwidth utilization may not be satisfied due to the dependency on the upstream subframe period, the upstream polling sequence, and the total number of active ONUs. One disadvantage of this scheme is that it is not suitable for delay-sensitive traffic due to longer queuing delay experienced in the OLT.

\section{Downstream Centric Scheduling (DCS) Algorithm}

The second scheduling policy presented in this paper is that the OLT stores downstream traffic in a First-In FirstOut (FIFO) buffer and the ONU has to be awake and 


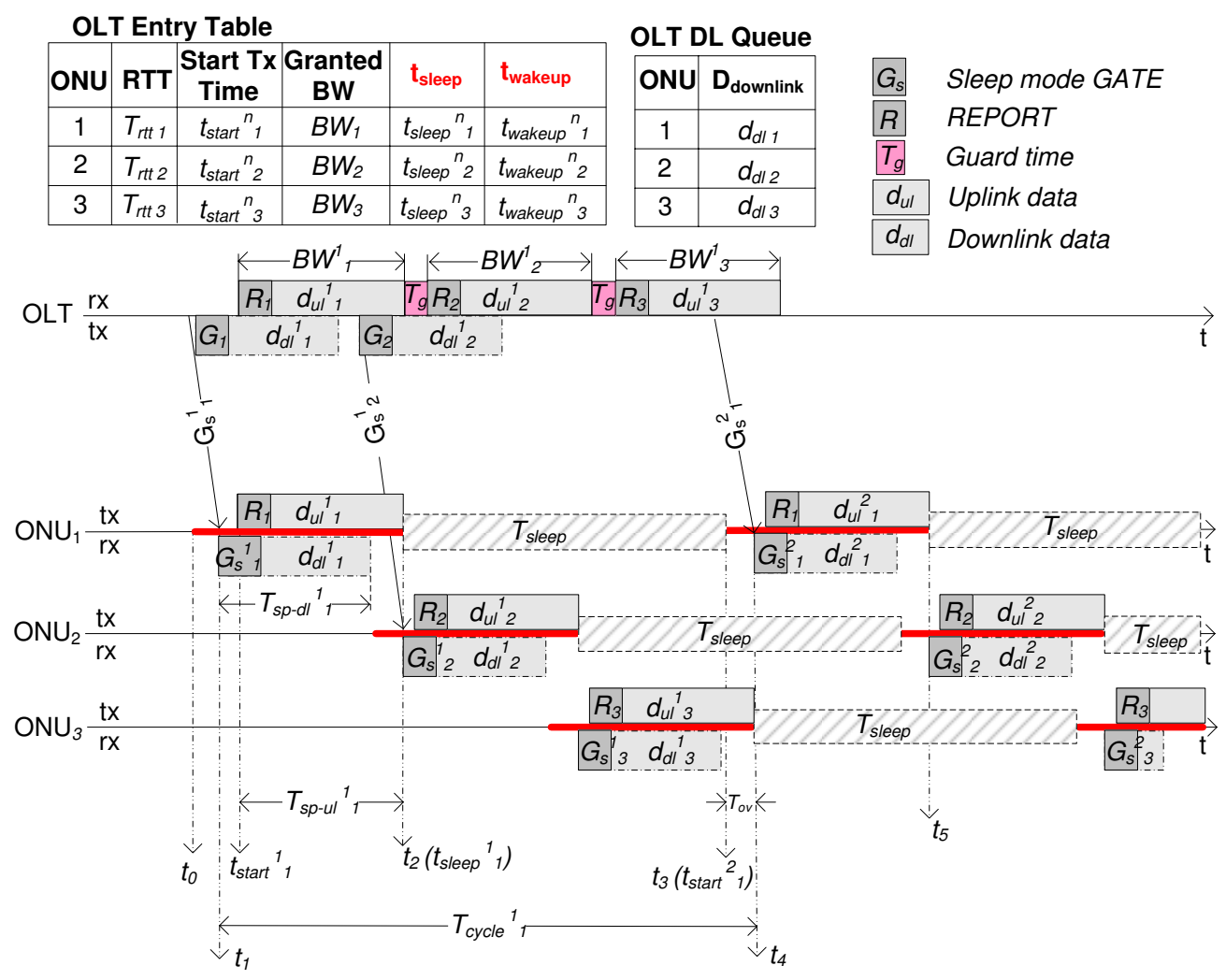

Figure 3. Sleep mode operation with Upstream Centric Scheduling (UCS) scheme.

receive downstream traffic whenever the OLT sends one. The awake periods are assigned to favor both upstream and downstream traffic. The DCS scheme is illustrated in Fig. 5. The sleep mode GATE message, $G_{s i}^{n}$, is used for both upstream bandwidth allocation and sleep period assignment. The upstream transmission period is calculated using the same equations $4-8$ as in the UCS scheme. For example, the $G_{s 1}^{1}$ at time $t_{1}$ indicates that the next polling time is at time $t_{8}$ and the $O N U_{1}$ needs to wake up at time $t_{7}$. In the downstream, the OLT serves data in a first-in first-out order. For instance, shown in Fig. 5, during the upstream subframe, $d_{u l 1} 1$, the OLT sends packets to both $O N U_{1}\left(\right.$ data $\left.d_{d l}^{1}\right)$ and $O N U_{2}\left(\right.$ data $\left.d_{d l 2}^{1}\right)$.

As shown in Fig. 5, the admission information for both upstream and downstream transmission is notified by the sleep mode GATE message. The $G_{s_{1}}^{1}$ informs $O N U_{1}$ the allocated upstream transmission of $d_{u l} 1$ and assigned sleep period during $t_{3}$ to $t_{7}$. After transmitting $d_{d l 2}^{1}$ to the $O N U_{2}$, the OLT gets data $d_{d l}{ }_{1}^{1}$ from the head of its downlink queue and sends to the $O N U_{1}$ together with $G_{s 1}^{2}$. Since there is data $d_{d l}^{2}$ in the buffer, the $O N U_{1}$ is notified to wake up at $t_{4}$ instead of $t_{7}$. The sleep period is assigned if there is neither upstream nor downstream transmission scheduled. If the OLT has queued data for the ONU, the wakeup time for the next data can be calculated. For example, the period between $t_{3}$ and $t_{4}$ is the idle period for $O N U_{1}$. Otherwise, the OLT sends a message to inform the ONU of remaining in awake mode in order to avoid missing any downstream traffic. In the following, the assignment of sleep period is discussed in details.

Under the condition that the downstream traffic terminates the sleep mode and the OLT assign the sleep period for the $i^{\text {th }} \mathrm{ONU}$, we distinguish four possibilities as shown in Fig. 6. The sleep period is determined by favoring both the successful reception of upstream and downstream data. Accordingly, we denote $G_{s}$ as the GATE control message carrying the information of sleep period, such as the start time of sleep period $\left(t_{\text {sleep }}\right)$ and the wakeup time $\left(t_{\text {wakeup }}\right)$. Let $T_{s p-u l_{i}^{n}}$ and $T_{s p-d l_{i}^{n}}$ be the $n^{\text {th }}$ subframe period of uplink and downlink transmission, respectively. In addition, the $T_{o v}$ period represents an overhead time for clock recovery and synchronization.

1) Case0: GATE0 is used to inform the allocated upstream bandwidth for the $O N U_{i}$. Because the OLT has full knowledge about the upstream bandwidth allocation, the sleep interval is determined, including both the sleep time $\left(t_{\text {sleep } 0}\right)$ and the wakeup time $\left(t_{\text {wakeup } 0}\right)$.

2) Case 1: The transmission of downstream data $\left(T_{s p-d l}^{1}\right)$ is completed within the allocated upstream subframe period. If there is other queued data in the OLT, such as the payload with GATE2, the sleep period is assigned as same as in Case0. If the next received data is the payload with GATE3, the time to wake up is reassigned as same as $t_{\text {wakeup } 2}$. If there is no more queued data in the OLT for the $O N U_{i}$, the original assignment, $t_{\text {sleep } 0}$, 


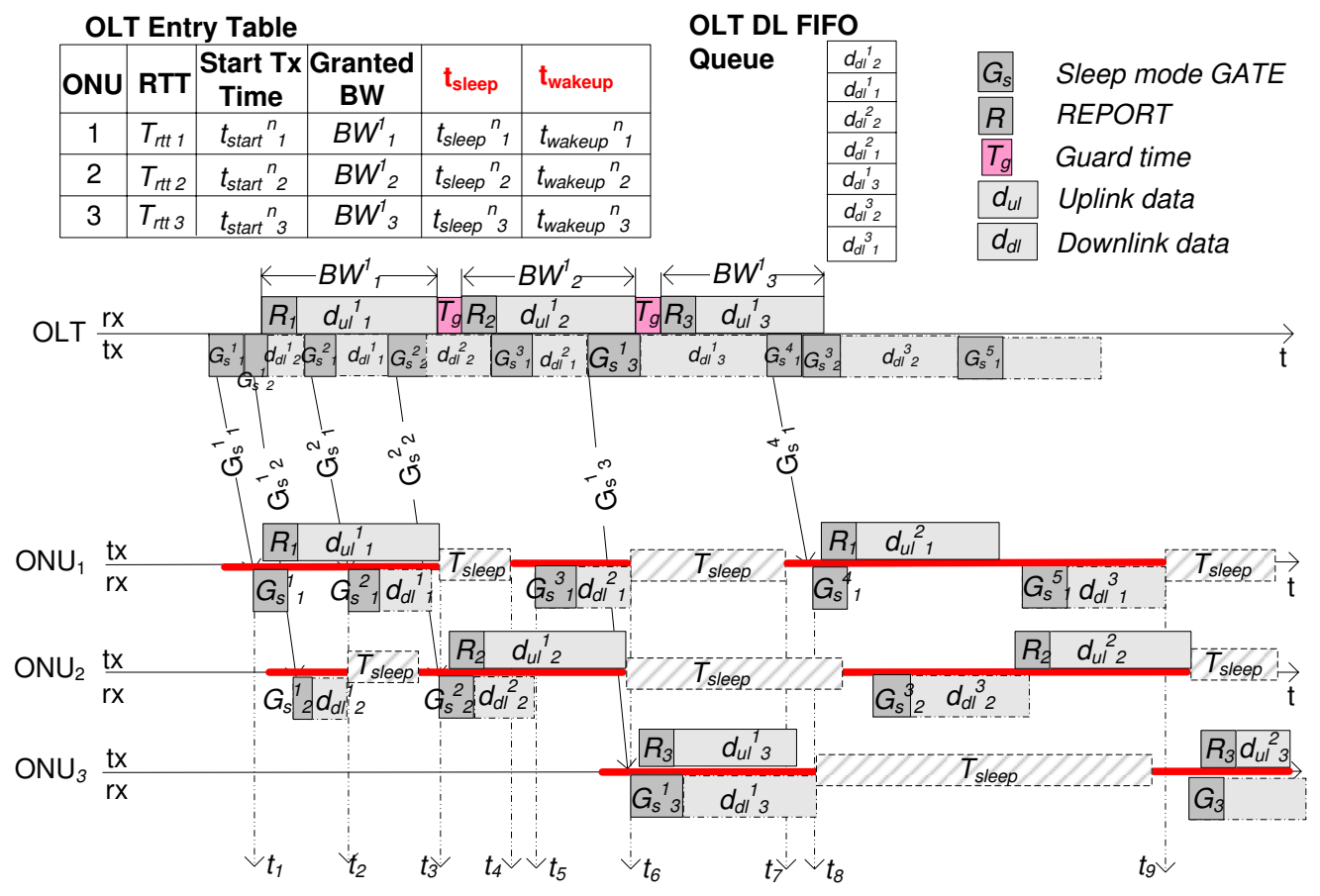

Figure 5. Sleep mode operation with Downstream Centric Scheduling (DCS) scheme.

is removed, because $O N U_{i}$ should keep awake in order to avoid missing future arrival data.

3) Case 2: In this case, the downstream data $\left(T_{s p-d l_{i}^{2}}\right)$ can not be finished before the start time of the sleep period as assigned in Case 0 and Case 1 . Therefore, the start time of sleep period is postponed to $t_{\text {sleep } 2}$. The OLT examines its downstream queue and schedules the transmission of the next down-

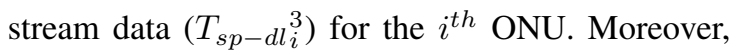
the wakeup time $\left(t_{\text {wakeup } 2}\right)$ is calculated in order to ensure a successful transmission.

4) Case 3: In this case, there is downstream data received during the sleep interval. In GATE3 control message, the new time of entering the sleep mode is updated, $t_{\text {sleep } 3}$, which is calculated based on the downstream subframe period $\left(T_{s p-d l_{i}^{3}}\right)$.

5) Case 4: As showing in the last GATE4 in this figure, there is downstream data arrived during the sleep interval and lasted till the next upstream subframe period. Upon the sleeping period is completed, the ONU transits into the awake mode and will receive the GATE4 message with the information of upstream bandwidth allocation, such as the start time and length of $T_{s p-d l}{ }_{i}$. Thus, the GATE4 message contains bandwidth assignments for both upstream and downstream subframe period.

The flowchart of the DCS algorithm implemented in the OLT is described in Fig. 7. Compared to the UCS algorithm, the OLT checks the available data in the buffer and update the sleep period for the destination ONU with a sleep mode GATE message. The wakeup time is precisely calculated and assigned, so that the ONU can be active to carry out the next upstream or downstream transmission. Since the operations of the above scheduling mechanism requires the OLT to locate the next packet in the buffer in order to calculate the wakeup time, ONUs which have no more queued packets cannot enter the sleep mode. In this case, the OLT assigns the sleep period as zero.

In order to calculate the total energy consumption in the DCS algorithm based EMM. We define the awake period for upstream transmissions $\left(T_{\text {awake-ul }}\right)$ and downstream transmissions $\left(T_{\text {awake-dl }}\right)$ separately. First, we compute the awake period and sleep period in the upstream direction (shown in Equation 10 and Equation 11), which is similar to Section IV-B.

$$
T_{\text {awake-ul,i }}^{n}=T_{s p-u l, i}^{n}+T_{o v}, \quad i \in K, n \in N
$$

$$
T_{\text {sleep-ul }, i}^{n}=T_{c y c l e, i}^{n}-T_{s p-u l, i}^{n}-T_{o v}, \quad i \in K, n \in N
$$

Next, assuming that there are $M$ downstream transmissions to the $i^{\text {th }}$ ONU. For the downstream, the awake period is calculated (in Equation 12) differently in each case mentioned as Fig. 6. In Case1, $O N U_{i}$ is in awake state, so that the awake period for receiving downstream data is zero. From Case2 to Case4, if the time for waking up and sleeping is assigned, the period that the ONU has to wake up from its sleep mode is calculated as the interval between $t_{\text {sleep }}$ and $t_{\text {wakeup }}$. Illustrated in Equation 13, the sleep period is the upstream sleep period minus the awake period used for the downstream transmission, which occurs during the upstream sleep period. 


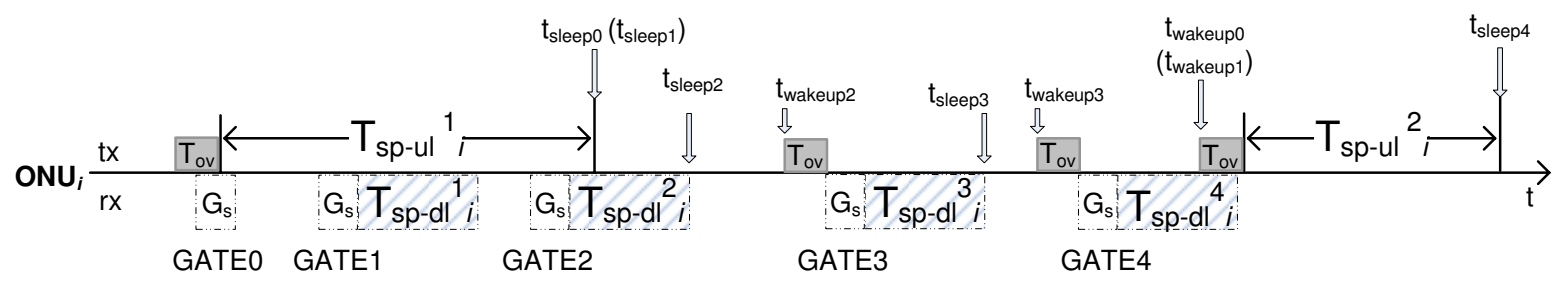

Figure 6. Downstream transmission in the sleep period model.

$$
\begin{array}{rlr}
T_{\text {awake-dl }, i}^{n} & =\sum_{m=1}^{M} T_{\text {awake-dl }, i}^{m} \\
& =\sum_{m=1}^{M} \begin{cases}0 & \text {, if Case1 } \\
t_{\text {sleep } 2}^{m}-t_{\text {sleep } 1}^{m} & \text {, if Case2 } \\
t_{\text {sleep } 3}^{m}-t_{\text {sleep } 2}^{m} & \text {, if Case3 } \\
t_{\text {sleep } 0}^{m}-t_{\text {sleep } 3}^{m} & \text {, if Case4 }\end{cases}
\end{array}
$$

$$
T_{\text {sleep-dl,i }}^{n}=T_{s p-u l, i}^{n}-\sum_{m=1}^{M} T_{\text {awake-dl, } i}^{m}, \quad i \in K, n \in N
$$

After analyzing the active behavior of an ONU in both the upstream and downstream transmissions, the total awake and sleep periods are concluded as Equation 14 and Equation 15:

$T_{\text {awake-total }, i}^{n}=T_{\text {awake-ul, },}^{n}+T_{\text {awake-dl, },}^{n}, \quad i \in K, n \in N$

$$
T_{\text {sleep-total }, i}^{n}=T_{\text {sleep-dl,i }}^{n}, \quad i \in K, n \in N
$$

The total energy consumed is calculated in Equation 16. The DCS sleep mode scheduler transmits downstream traffic in a flexible way, unlike in the UCS that only the active ONU scheduled with an upstream transmission period can receive data from the OLT. The OLT tries to schedule the queued downstream traffic in a way to minimize the delay. The UCS is simple where the sleep period and wakeup time is determined only by the upstream bandwidth allocation. On the other hand, the DCS scheduler requires to keeping track of both downstream and upstream transmission windows.

$$
\begin{aligned}
\boldsymbol{E}_{\mathbf{D C S}} & =\sum_{i=1}^{K} \sum_{n=1}^{N}\left[T_{s p-u l, i}^{n} \cdot P_{\text {awake }}\right] \\
& +\sum_{i=1}^{K} \sum_{n=1}^{N}\left[T_{\text {sleep }- \text { total }, i}^{n} \cdot P_{\text {sleep }}\right]
\end{aligned}
$$

\section{Simulation Results}

\section{A. Simulation Scenario}

In this section, we study performances and features of our proposed energy management mechanism compared to the fixed power control, which is represented as Power Ignoring Scheme (PIS). Simulations have been carried out by means of OPNET network simulator [19] to measure the performance metrics. The performed simulations aimed to show the diverse effects of system parameters, such as the upstream scheduling scheme, the number of connected ONUs, and the arrival packet size. Then the benefits that can be obtained while utilizing the proposed EMM in EPON. The following parameters are monitored, such as the power consumption, the network throughput, and the average queuing delay. The average awake time in ONUs is used as a merit for energy efficiency performances. The less time an ONU is awake, the less energy an ONU consumes.

We have assumed that the OLT is connected to $K$ ONUs ( $K=16$ or 32$)$, which are enabled with power saving functionality. The optical link rate is $1 \mathrm{Gbps}$ for both upstream and downstream transmission. The guard time between two consecutive transmission slots is 5 us. For allocating upstream bandwidth among ONUs, both fixed scheme (e.g. TDM) and dynamic scheme (e.g. IPACT) are implemented at the OLT. The maximum transmission cycle is set to $2 \mathrm{~ms}$. As the proposed power saving mechanism with two scheduling schemes cooperates the optical downstream bandwidth scheduler at the OLT, it is reasonable to monitor the performance of downstream traffic. Traffic is generated following Poisson distribution and the length of packets is generated independently between 64 bytes to 1200 bytes. Destinations of downstream traffic are uniformly distributed among connected $K$ ONUs. The MPCP control messages, GATE and REPORT, are formatted according to the IEEE 802.3ah specification. The sleep mode GATE message, $G_{s}$, is an extension of the standard GATE message. The additional two 4 bytes fields are embedded as assigned sleep time and wakeup time. The total size of $G_{s}$ message is set to 41 bytes.

\section{B. Validation of EMM with the fixed upstream bandwidth allocation}

In Section IV, we analyze the energy efficiency mechanism with two proposed scheduling approaches and derive the consumed power accordingly. The simulation scenario consists of 16 ONUs and the OLT grants fixed upstream bandwidth to each ONU. The results in Fig. 8 refer to the system behavior in the presence of an fixed upstream bandwidth allocation scheme deployed at the OLT. They show the energy consumption, represented by the ONU 


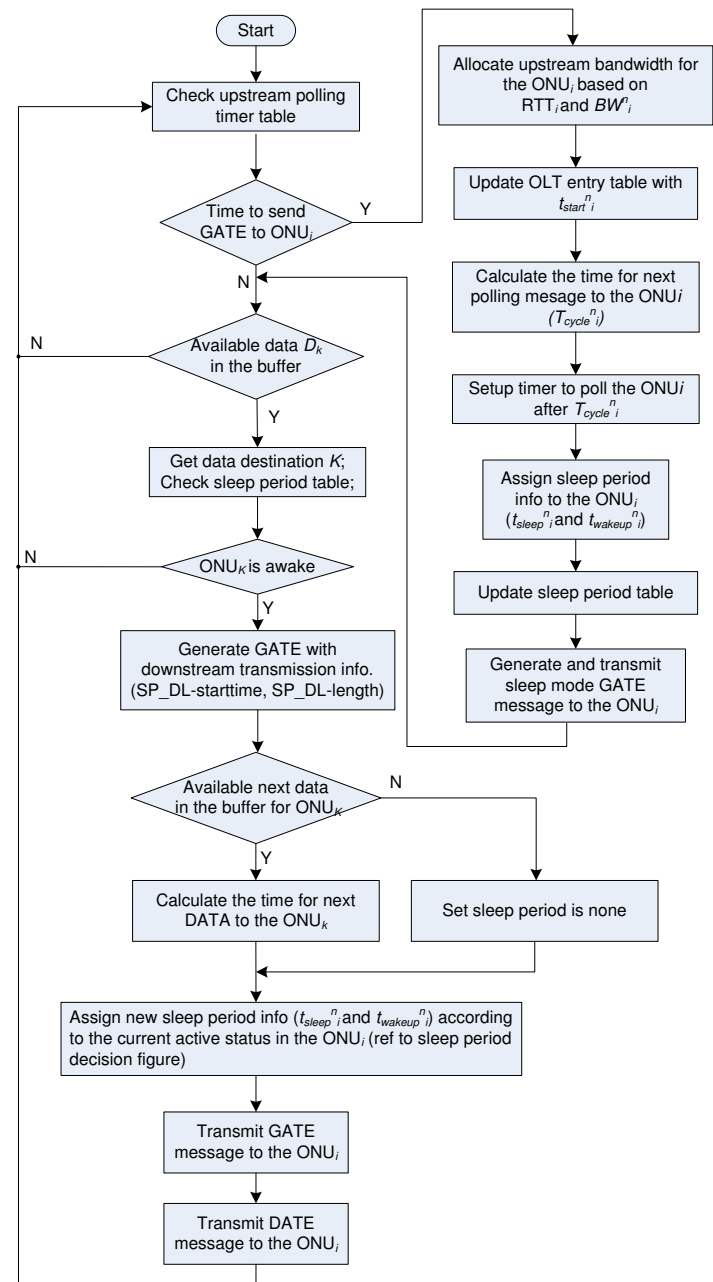

Figure 7. Flow chart of the Download Centric Scheduling (DCS) Scheme.

awake time, when the downstream traffic load changes. In the case of PIS, ONUs constantly stay in awake state and consumes high energy ( $100 \%$ of the observation period). When the EMM is enabled in the EPON system, the awake time maintains at a low average percentage value if the Uplink Centric Scheduling (UCS) scheme is applied. As explained in Section IV-B, the period of being power on is decided by allocated upstream transmission periods in the UCS case. When each ONU is allocated with fixed amount of upstream bandwidth, the awake time is also fixed. On the other hand, under the DCS case, ONUs wake up and communicate with the OLT for either upstream or downstream traffic. When arrival traffic is less than the optical output link rate, the awake time of EMM-DC is slightly increased due to the additional active period for downstream traffic. When the arrival traffic rate is high, the awake time increases and close to the level as in the PIS case. With respect with energy efficiency, the proposed energy management mechanism outperforms the traditional power ignoring mechanism. Particularly, using the uplink centric scheduling scheme saves up to $90 \%$ power on time. The energy consumption in the DCS case varies when the traffic load changes.
Fig. 9 shows the network throughput under the PIS and EMM cases. The downstream throughput is monitored, because the upstream throughput is not affected by the proposed power saving mechanism. When the EMM is deployed, both DCS and UCS schemes degrade the throughput performance. In the DCS case, throughput is decreased due to the additional overhead by introducing the sleep mode GATE control message. Because the wakeup time and sleep time are assigned to ONUs when they receive every downstream packet, it causes a portion of control message overhead. In the UCS case, the network throughput is reduced greatly. The reason is that the OLT can dispatch downstream traffic during its allocated upstream transmission period. Assuming the OLT polls and grants all ONUs in a Round Robin (RR) order, each ONU can derive one over sixteen percent of total downstream link capacity. The link utilization factor, computed as the amount of traffic delivered to the destination successfully over the total link capacity in the simulation time. The higher the network throughput, the high link utilization. The proposed EMM receives similar results compared to the PIS case, when the DCS scheme is applied. However, performance is highly downgraded in the UCS scheme.

The average queueing delay for analyzing performance differences under different power saving settings is shown in Fig. 10. The average delay of the EMM DC scheme is better than that of the UCS scheme since, with UCS scheme, downstream packets have to wait for their awake period in the OLT buffer. We observe that when the traffic intensity is low, average delay remains constant since the transmission rate is high enough to serve under a range of values of traffic load. However, when the incoming traffic intensity reaches a certain point and causes congestion, average delay increases rapidly. As expected, the average queueing delay is consistent with the throughput performance, where the EMM DC scheme achieves better performance.

\section{Impact of Upstream Bandwidth Allocation Schemes}

Now we examine the impact of upstream bandwidth allocation schemes on the proposed energy management mechanism with the uplink centric scheme. In this scenario, only upstream traffic from ONUs to the OLT is considered to highlight the results differed from different upstream bandwidth allocation schemes. If the downstream traffic is involved, then additional time is required for transmitting downstream packets.

Fig. 11 illustrates a simple scenario that shows the relationship between the upstream bandwidth allocation algorithm, TDM and DBA, with the ONU awake time. Because the downstream traffic is ignored, the sleep period is assigned to each ONU only based on the allocated upstream bandwidth, as the EMM UCS performs. In the TDM case, each ONU is assigned with a fixed slot time. In the DBA scheme case, the OLT grants ONUs according to their bandwidth requests. Under low traffic load, buffered data at ONUs are small, and therefore, 


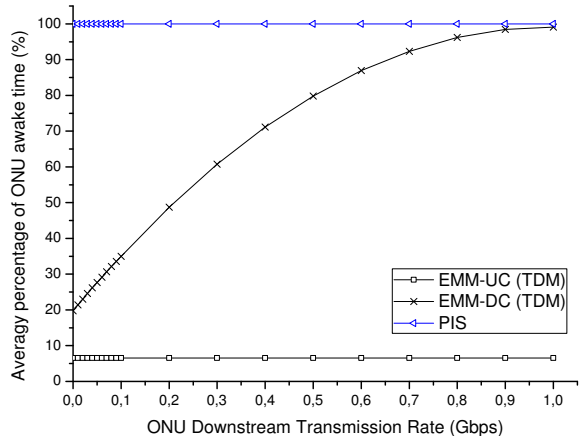

Figure 8. Average ONU awake time in PIS, EMM-UC, and EMM-DC algorithms.

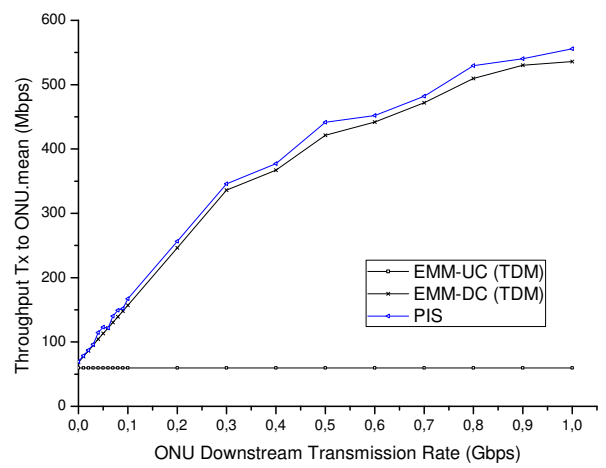

Figure 9. Network throughput in PIS, EMM-UC, and EMM-DC algorithms.

small amount of requested time slots. The percentage of awake time is increased along with the increased traffic rate. Because the maximum upstream slot time is constrained as $2 \mathrm{~ms}$, the DBA based approach has similar results under the heavy traffic load.

Fig. 12 and Fig. 13 show the network throughput and queuing delay for the downstream traffic, respectively. The volume of downstream traffic is fixed at 1 Gbps and destination is uniformly distributed among 16 ONUs. Consistent with the curve of awake time, the downstream throughput increases when the ONU receives high input traffic and requests for large timeslots. On the other hand, when the required upstream transmission period is small, ONUs are turned off most of the observation time and downstream data are queued at the OLT. Thus the queuing delay is high under light traffic load in the dynamic bandwidth allocation case.

From previous studies [18], dynamic bandwidth allocation schemes improve EPON channel utilization and provide QoS guarantee. Our simulations over the upstream channel have demonstrated that the dynamic upstream bandwidth allocation scheme gains system power efficiency. However, the delay performance is downgraded when the upstream traffic load is low.

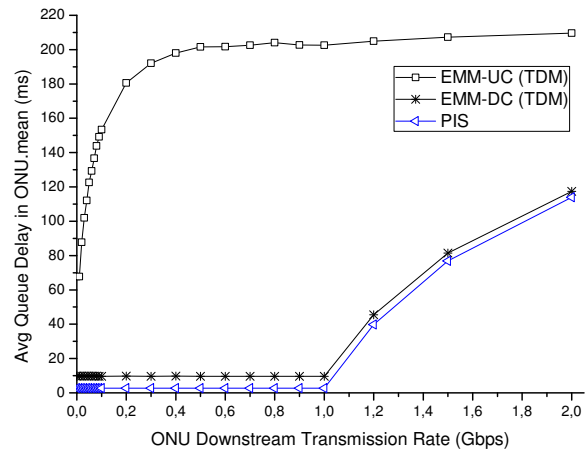

Figure 10. Average queueing delay in PIS, EMM-UC, and EMM-DC algorithms.

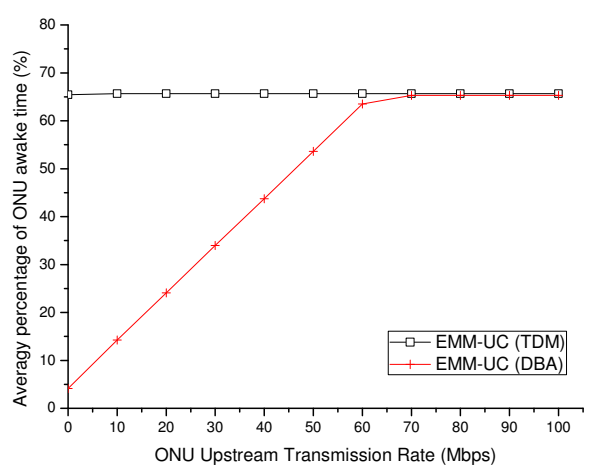

Figure 11. Awake time compared in different upstream bandwidth allocation schemes.

\section{Impact of the sleep mode GATE message overhead}

In this study, simulations show impacts of the sleep mode GATE control message. The MPCP control messages, GATE and REPORT, are formatted according to the IEEE 802.3ah specification. The sleep mode GATE message, $G_{s}$, is an extension of the standard GATE message. The additional two 4 bytes fields are embedded as assigned sleep time and wakeup time. The total size of $G_{s}$ message is set to 41 bytes. In the UCS case, the sleep mode GATE message is used to poll ONUs, grant upstream bandwidth, and inform assigned sleep period. The Gs is generated and sent to ONUs in the same means as the original MPCP case. However, in the DCS case, $G_{s}$ message is transmitted together with each downstream packet, which results in amount of control message overhead. Therefore, in this test case, we simulate a network scenario with 16 ONUs and focus on EMM DCS scheme.

Fig. 14 shows the number of transmitted $G_{s}$ as a function of incoming packet sizes. In all cases, the total number of $G_{s}$ is increased at the downstream data rate increases. The size of data packets are varied between 400 bits and 9400 bits. Under a certain input data rate, the larger the data size, the less transmitted $G_{s}$ control message. With respect to bandwidth utilization, increased number of $G_{s}$ message introduces high value of control 


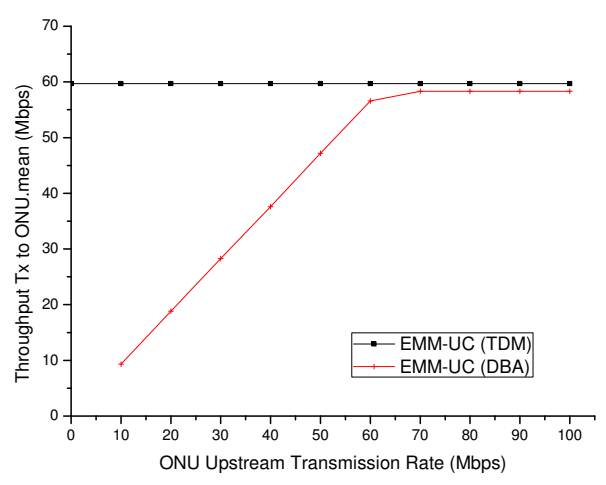

Figure 12. Network throughput compared in different upstream bandwidth allocation schemes.

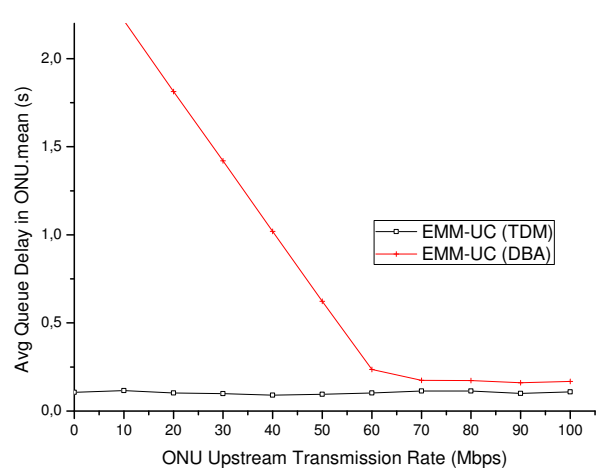

Figure 13. Average queuing delay compared in different upstream bandwidth allocation schemes.

overhead, therefore, results in low bandwidth utilization.

The delay performance is illustrated by Fig. 15. The delay difference is hardly to tell when the downstream traffic load is low. When traffic load becomes high, the large packet size case produces better delay performance. This is for the reason that higher channel utilization can be achieved by reducing the ratio of the overhead of $G_{s}$ message and the size of payload.

\section{E. Performance comparison with multiple ONUs}

We now consider a scenario where 32 ONUs are connected to the OLT. With this network structure, an ONU is allocated less upstream transmission periods. Fig. 16 shows the awake periods are reduced in both UCS and DCS schemes compared to the 16 ONUs structure. Consequently, the queuing delay is increased (shown in Fig. 17). When the traffic load becomes high enough, the downstream traffic takes all transmission time and consumes the same power as in the PIS case.

\section{CONCLUSION}

In this paper, we focus on the energy consumption in the EPON system. We proposed and investigated an analytical model for the energy expenditure in EPON. We developed an energy management mechanism for optimal assignments of sleep periods to ONUs. The delay constraints that need to be satisfied is analyzed in the

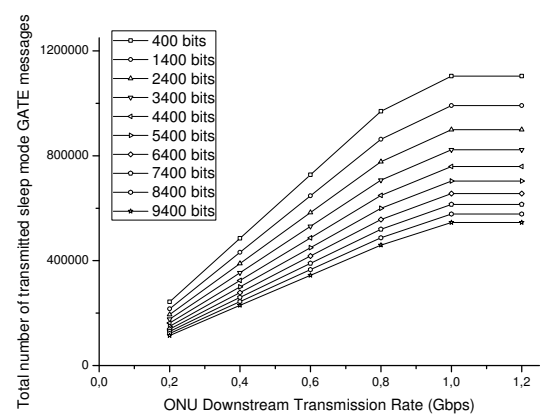

Figure 14. the number of transmitted $G_{S}$ as a function of incoming packet sizes.

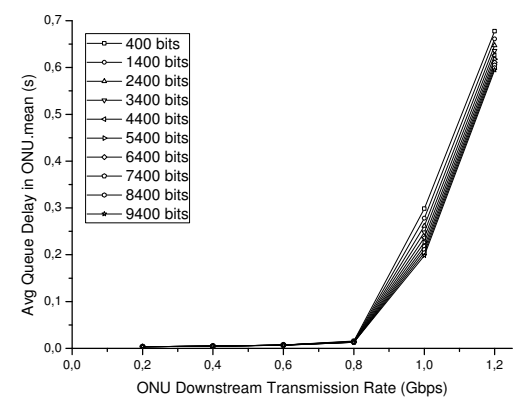

Figure 15. Average queuing delay performance as an impact of the sleep mode GATE message overhead.

scheduling problem. We solve the problem by proposing two heuristic scheduling algorithms: the upstream centric scheduling algorithm and the downstream centric scheduling algorithm. An EPON system with energy efficiency based scheduling schemes are simulated in the OPNET simulator. We verified the solution quality with respect to a set of chosen metrics such as awake time and queuing delay. Specifically, we compared the energy performance and the network performance under different traffic load and different upstream bandwidth allocation schemes. We found that the energy management mechanism reduces the power consumption, and the DCS algorithm outperforms the UCS algorithm in the delay performance.

\section{REFERENCES}

[1] C. Lange and A. Gladisch, "Energy consumption of telecommunication networks: A network operator's view," in Work shop on Optical Fiber Communication Confence (OFC) "Energy Footprint of ICT: Forecasts and network solutions", 2009.

[2] C. Lange, D. Kosiankowski, C. Gerlach, F. Westphal, and A. Gladisch, "Energy consumption of telecommunication networks," in 35th European Conference on Optical Communication (ECOC), 2009, pp. 1-2.

[3] M. Gupta and S. Singh, "Greening of the internet," ACM SIGCOMM, pp. 19-26, 2003.

[4] J. Baliga, R. Ayre, K. Hinton, W. V. Sorin, and R. S. Tucker, "Energy consumption in optical IP networks," IEEE/OSA Journal of Lightwave Technology (JLT), vol. 27, pp. 2391-2403, 2009. 


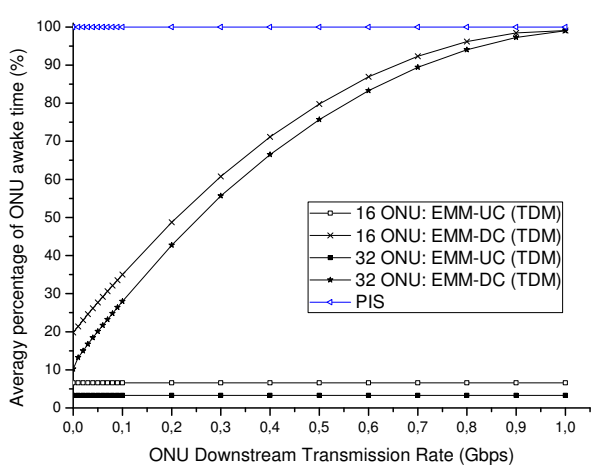

Figure 16. Awake time compared in scenarios with different connected AGs.

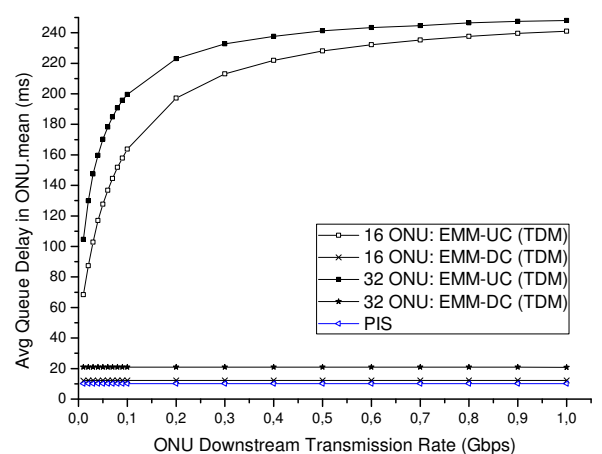

Figure 17. Network throughput compared in scenarios with different connected AGs.

[5] K. Christensen, C. Gunaratne, B. Nordman, and A. George, "The next frontier for communications networks: Power management," Computer Communications, vol. 27, pp. 1758-1770, 2004.

[6] C. E. Jones, K. M. Sivalingam, P. Agrawal, and J. C. Chen, "A survey of energy efficient network protocols for wireless networks," Wireless Networks, vol. 7, pp. 343358, 2001.

[7] J. Lee, C. Rosenberg, and E. Chong, "Energy efficient schedulers in wireless networks: Design and optimization," Mobile Networks and Applications, vol. 11, pp. 377-389, 2006.

[8] Y. Zhang, "Performance modeling of energy management mechanism in IEEE 802.16e mobile WiMAX," in Wireless Communications and Networking Conference (WCNC), 2007, pp. 3205-320.

[9] R. Cohen and B. Kapchits, "An optimal wake-up scheduling algorithm for minimizing energy consumption while limiting maximum delay in a mesh sensor network," IEEE/ACM Transactions on Networking, vol. 17, pp. 570$581,2009$.

[10] J. Hsieh, T. Lee, and Y. Kuo, "Energy-efficient multipolling scheme for wireless LANs," IEEE Transactions on Wireless Communications, vol. 8, pp. 1532-1541, 2009.

[11] Y. Chen and S. Tsao, "Energy-efficient sleep-mode operations for broadband wireless access systems," in IEEE Vehicular Technology Conference, 2006, pp. 1112-1116.

[12] T. Smith, R. S. Tucker, K. Hinton, and A. V. Tran, "Implications of sleep mode on activation and ranging protocols in PONs," 21st Annual Meeting of the IEEE Lasers and Electro-Optics Society (LEOS), vol. 11, pp. 604-605, 2008.

[13] S. Wong, L. Valcarenghi, S. Yen, D. Campelo, S. Yamashita, and L. Kazovsky, "Sleep mode for energy sav- ing PONs: Advantages and drawbacks," in Globlecom'09 Second International workshop on Green Communication, 2009.

[14] R. Kubo, J. Kani, Y. Fujimoto, N. Yoshimoto, and K. Kumozaki, "Proposal and performance analysis of a powersaving mechanism for 10 gigabit class passive optical network systems," in NOC, vol. 1, 2009, pp. 87-94.

[15] EPON Powersaving via Sleep Mode, IEEE 802.3az Meeting, 2008

[16] I. Cerutti, L. Valcarenghi, and P. Castoldi, "Power saving architectures for unidirectional WDM rings," in Optical Fiber Communication (OFC), 2009, pp. 1-3.

[17] http://www.ieee802.org/3/efm/, IEEE 802.3ah Ethernet in the First Mile Task Force.

[18] K. Glen, M. Biswanath, and P. Gerry, "IPACT: a dynamic protocol for an Ethernet PON (EPON)," IEEE Communication Magazine, vol. 40, pp. 74-80, 2002.

[19] http://www.opnet.com/, OPNET Modeler 14.5, 2005.

Ying Yan received the B.Eng. degree in electrical engineering from the Beijing University of Technology, China, in 2002 and the M.S. degree in electronics engineering in 2004 from the Technical University of Denmark. After completing the Master degree, Ying Yan began to pursuit her Ph.D. studies on telecommunication engineering in 2005. During 2006-2007, she worked as a research scientist at the department of communication platforms in the Technical Research Centre of Finland (VTT), Finland. While pursuing the Ph.D. study, Ying Yan participated in European projects (the IST-MUPBED project and the ICT-ALPHA project) and a Danish national project (the HIPT project). Currently she is an Assistant Professor at the Technical University of Denmark. Her research interests include scheduling algorithms, resource management mechanism, quality of service, and energy efficiency in fixed wireless networks and Carrier Ethernet transport network.

Lars Dittmann was born in 1962 and received the M.Sc. $\mathrm{EE}$ and $\mathrm{Ph} . \mathrm{D}$. from the Technical University of Denmark in 1988 and 1994, and is currently professor at the university within the area of integrated networks. He has since January 1999 been heading the network competence area (covering both optical and electrical networks) within DTU Fotonik at the Technical University of Denmark and was prior to that responsible for electronic switching and ATM networks at the Center for Broadband Telecommunication. Main research area is network architectures and performance. 